\section{A TYPICAL FEATURES IN IRVAN SYNDROME DEMONSTRATED ON MULTIMODAL IMAGING}

\author{
Hashim Ali Khan, OD, FAAO, \\ (Corresponding Author) \\ SEHHAT Foundation Hospital, Main KKH, Danyore, \\ Gilgit, Pakistan
}

\section{Muhammad Aamir Shahzad, MBBS, FRCS \\ Aziz Fatimah Medical and Dental College, Faisalabad, Pakistan}

Muhammad Amer Awan, FRCS, FRC Ophth

Shifa International Hospital, Islamabad, Pakistan

\section{Abstract:}

Purpose: The purpose of this work was to describe idiopathic retinal vasculitis, aneurysms, and neuroretinitis (IRVAN) syndrome in a 32-year-old male.

Methods: Case report.

Case report: A 32-year-old male presented with acute visual disturbance. Multimodal imaging revealed retinal vasculitis, aneurysms and neuroretinitis in addition to vitreous inflammation, retinal ischemia and epiretinal membrane. Optical coherence tomography angiography (OCTA) demonstrated multiple microaneurysm in both eyes.

Conclusion: Multimodal imaging was helpful in diagnosing and differentiating IRVAN syndrome from other similarlooking clinical entities. Retinal aneurysms and retinal ischaemia were detectable by fundus fluorescein angiography (FFA). However, OCTA showed multiple microaneurysms that were not clearly differentiable by FFA because of vitreous inflammation and vascular leakage .Microaneurysms in IRVAN syndrome area previously unreported observation. OCTA may help detect thesechanges with accurate details.

Keywords: IRVAN syndrome; Multimodal imaging; Neuroretinitis; OCTA in IRVAN syndrome; Retinal Vasculitis.

\begin{tabular}{|l|l|}
\hline \multicolumn{2}{|c|}{ Access this article online } \\
\hline Quick Response Code: & $\begin{array}{l}\text { Website: } \\
\text { https://ophthalmolcases.com/index. } \\
\text { php/hat }\end{array}$ \\
\cline { 2 - 2 } & $\begin{array}{l}\text { DOI: } \\
\text { 10.30546/2788-516X.2021.2.1.5 }\end{array}$ \\
\hline
\end{tabular}

\section{Introduction}

Idiopathic retinal vasculitis, aneurysms, and neuroretinitis (IRVAN) is a rare spectrum of ocular changes characterized by vasculitis, arteriolar aneurysms, and neuroretinitis affecting otherwise healthy individuals in the 2nd to 4 th decade of lifewith a female predilection. The retinal changes can be preceded by anterior uveitis and vitritis. The clinical presentation and course vary remarkably.The disease may have a self-limiting course in some eyes, while many eyes progress to retinal oedema, ischaemia, and neovascularizationadvancing to neovascular glaucoma.1,2

The diagnosis of IRVAN syndrome is based on the presence of three major criteriathat are not caused by any other disease: retinal vasculitis, aneurysms, and neuroretinitis. Three minor and optional criteria are macular exudation, capillary nonperfusion (CNP) and retinal neovascularization. 1,2

Retinal vasculitis is characterized by segmented or confluent perivascular exudation/sheathing that can be primary in nature or secondary to a wider variety of ocular and systemic associations. It is best depicted by FFA as leakage of dye and staining of vessel walls corresponding to the extent of vascularinvolvement.1,2

The location, size and distribution of aneurysms in IRVAN syndrome are extremely dynamic, but there tinal arteriolar network and optic nerve head are usually involved. Typically, the aneurysms appear as saccular or fusiform out pouchings or as tied-knot lesions of arterial trees. Optic disc aneurysms are defined as pathognomonic and are sometimes the earliest sign of IRVAN syndrome.1 The mechanisms underlying the development of aneurysms include focal damage and weakening of vascular segments leading to fusiform and saccular ectasia at weaker segments due to hydrostatic pressure. Chawla et al.s proposed a congenital or developmental origin of aneurysmal changes in IRVAN syndrome; however, most authors believe the changes are mediated by inflammation. Nonetheless, the acquired nature, mild intraocular inflammation, resolution of aneurysms over time or in response to anti-inflammatory therapy and immune modulators all support an inflammatory origin.1,2,4

Neuroretinis in IRVAN syndrome is characterized by hyperaemic and oedematous optic discs and is depicted by FFA as a hyperfluorescent disc in the later phase of angiogram 1. Other ocular features include macular oedema, epiretinalmem branes (ERMs), optic disc aneurysms, retinal capillary nonperfusion (CNP), retinal vascular occlusions, retinal neovascularization, vitreous haemorrhage and iris rubeosis.1,2 
Low-grade inflammatory activity is usually seen in the anterior chamber and/or vitreous at some point during the disease course. Numerous uveitides, retinal vasculopathies and systemic diseases mimic IRVAN syndrome and need to be differentiated.

Often, a case with an a typical presentation may pose a significant clinical challenge to the clinician in establishing a correct diagnosis. Multimodal imaging can be of great value in establishing a correct diagnosis.

Fundus fluorescein angiography (FFA) is an important tool in the evaluation of vasculitis, retinal oedema, aneurysms, and neuroretinitis. Areas of retinal ischaemia, proliferation and other vascular changes can be easily demarcated with FFA.

Optical coherence tomography angiography (OCTA) is a newly developed and emerging vascular imaging technique that helps study individual vascular networks of the retina and choroid. Although OCTA cannot detect retinal vasculitisand leaky blood vessels, it is beneficial in evaluating the actual vascular changes that are otherwise obscured on FFA scans by dye leakage from inflamed/leaky vasculature.5Here, we report and discuss the multimodal imaging features of IRVAN syndrome in a case with bilateral involvement. We describe a new finding that has not been reported before.

\section{Case Reports}

A 32-year-old, otherwise healthy male presented with a history of reduced vision in the OD over the past3 weeks. Past ocular and other medical histories were unremarkable. There was no family history of ocular or other systemic diseases. In addition to a comprehensive review of systems by an internist, a widerange of laboratory tests for common infectious and inflammatory uveitides were ordered. The workup algorithmand tests have been described elsewhere in the literature2.

All investigations were within normal limits. All inflammatory markers, infectious disease labs, and workups for systemic associations were normal. BCVA recorded 6/24 OD and 6/7 OS. Both pupils were equally round and reactive to light and near reflex. Anterior segments were unremarkable.

DFE was consistent with +2 and +1 vitritis OD and OS, respectively. A small resolving vitreous haemorrhage OD wasalso noted. Both optic discs were hyperaemicand inflamed; mixed retinal vasculitis (phlebitis and arteriolitis) was evident OU. (Figure 1)

FFA was able to delineate peripheral CNP and leaky hyperfluorescent optic discs. The severityand extent of retinal vasculitis were more remarkable on FFA scans than on clinical examination. (Figure 2)

Figure 1: Wide-field colour and red-free images showing retinal vasculitis and inflamed optic discs. Peripheral intraretinal haemorrhages $(B, D)$ can be seen $O S$.








Figure 2: Fluorescein angiography shows multiple bilateral aneurysms, CNP, and leakage from inflamed vessels and optic discs.Central 60 degrees of the retina $(A \mathcal{E} B)$ and peripheral view of $O D(C)$ and $O S(D)$.

Macula centred 3x3 mm OCTA with Angiovue, RTVue XR Avanti (Optovue, Fremont, CA),depicted several aneurysmal outpouchings of capillaries and arterioles in superficial and deep vascular plexuses OU. Aneurysms were more numerous and more obvious on OCTA scans than onFFAscans. In addition, the right eye showed an epiretinal membrane and macular thickening with cystoid spaces on structural OCT B-Scans. (Figure 3)
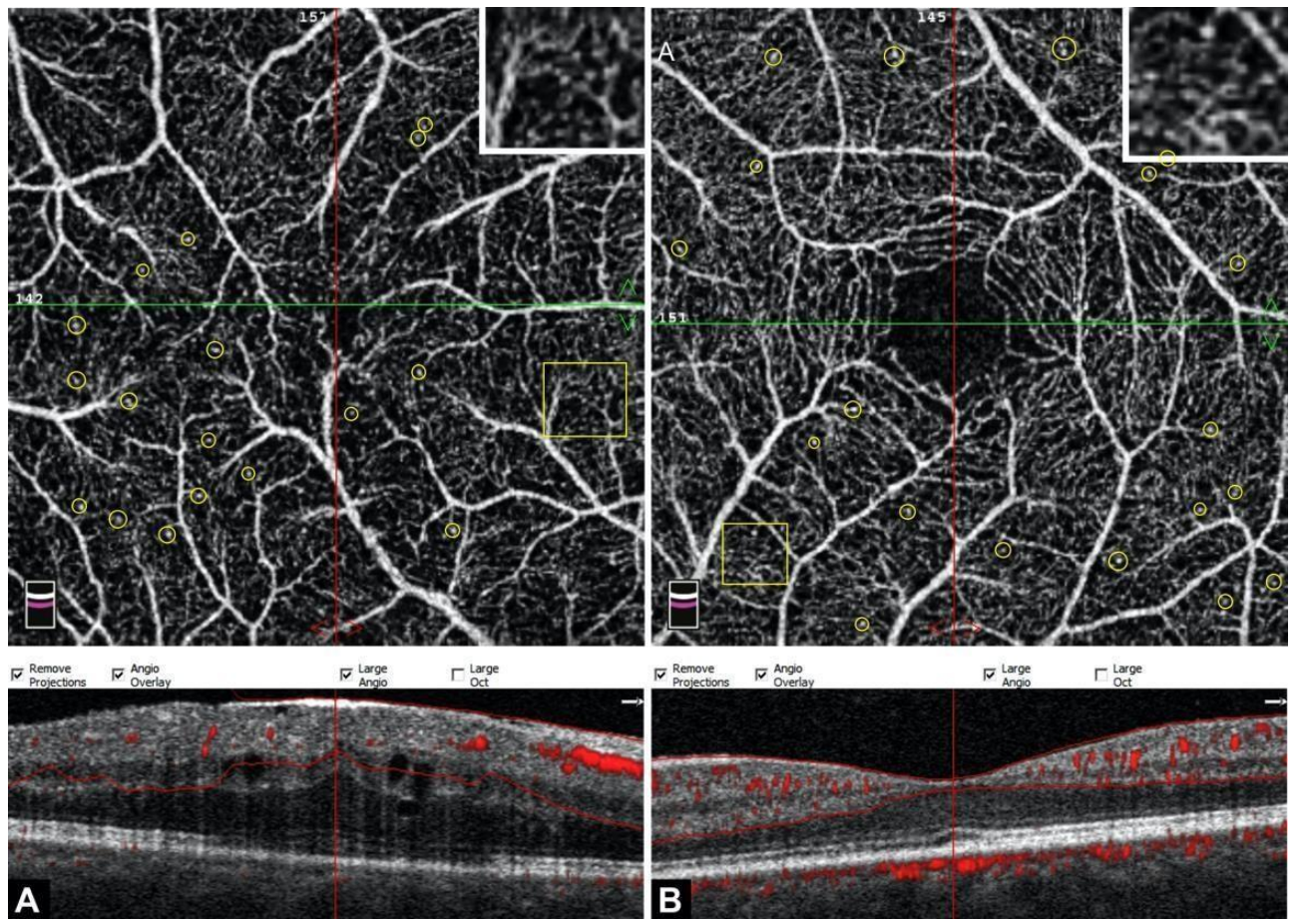

Figure 3: Composite OCTA of superficial and deep vascular networks (ILM-OPL) from both eyes. Multiple aneurysmal changes and corkscrew-like vascular alterations on the macula of both eyes are depicted by OCTA $(A, B)$. Structural OCT showing ERM, retinal thickening and cystoid changes $O D(A)$ and normal foveal morphology $O S$ (B). The inset shows an enlarged segment of the image, and some of the microaneurysms are encircled. 


\section{Discussion}

IRVAN syndrome is a rare and relatively poorly understood spectrum of ocular pathologies. It is a diagnosis of exclusion, and a wide range of ocular and systemic entities that may cause vasculitis, neuroretinitis and aneurysmal changes should be ruled out.Early diagnosis and treatment have a key role in visual outcomes.

Several therapeutic options exist, including systemic steroids, immune modulators, intravitreal vascular endothelial growth factor suppression, laser photocoagulation and intravitreal steroid implants. Multimodal imaging is of great value in gauging severity and for the selection of treatment approaches.

Retinal vasculitis is one of the key features seen in eyes with IRVAN syndrome. In most cases, it is well discernible on clinical examination. However, it may be more widespread and extensive on FFA scans than in the clinical evaluation.1 Retinal vasculitis in IRVAN syndrome is predominantly arteritis and arteriolitis; however, phlebitis has also been described in many cases.1,6

Both arterial and venular inflammation was ascertained in our patient. From a review of fundus images and descriptions in the published literature, we found that most eyes depict mixed retinal vasculitis involving both arteriolar and venular trees.1,3,4

Due to macular oedema in eyes with IRVAN syndrome, subtle retinal changes are difficult to observe clinically. An epiretinal membrane in the right eye of our patient was barely noticeable because of vitreous haze and altered retinal morphology secondary to macular oedema.

OCT was able to demarcate the retinal thickness and extent of ERM and its relationship to the fovea. ERM has been rarely described in IRVAN syndrome and suggests a possible inflammatory origin of the disease 1.

In most cases, FFA can help evaluate retinal vasculitis, aneurysms, optic disc leakage and areas of retinal ischaemia quite well. Our patient did not demonstrate any aneurysms on clinical examination or fundus images.

However, FFA was able to clearly delineate a few aneurysms and retinal ischaemia. Nevertheless, the observation of retinal aneurysms may be obscured by dye leakage from inflamed vasculature and vitreous activity.7 In the reported case, FFA was able to depict retinal arteriolar aneurysms.

Very few studies have described OCTA features in IRVAN syndrome. Marchese A, et al.8 described widefield OCTA for documenting areas of retinal ischaemia and other features of IRVAN syndrome.

Optic disc aneurysms, vascular alterations and aneurysmal changes in published by other groups.3,9
In our patient, OCTA clearly demonstrated multiple microaneurysms at retinal arterioles and capillary networks and corkscrew-like vascular alterations.

Nonetheless, to the best of our knowledge, microaneurysms involving macular capillary beds are an unreported observation. Capillary and arteriolar microaneurysms may be an uncommon feature or part of the evolution of IRVAN syndrome, as many authors have described a diverse pattern and course of aneurysms in this entity.10

These findings may be an early manifestation of the aneurysmal changes associated with IRVAN syndrome and may progress further or regress during the course of the disease. Alternatively, microaneurysms may be another component of the spectrum of vascular changes associated with IRVAN syndrome 10.

Although FFA has greater sensitivity than OCTA in detecting microaneurysms, the details may be obscured on FFA scans by leakage and oedema. Nonetheless, leakage and oedema do not obscure the actual changes invascular beds. 5

Our report describes the role of imaging in establishing an appropriate diagnosis. If FFA and OCTA were not acquired, the patient would have been diagnosed with any other spectrum of retinal vasculitis because the aneurysmal changes and areas of retinal ischaemia were not readily noticeable on clinical evaluation and fundus imaging.

\section{Conclusion}

IRVAN syndrome is a rare spectrum of retinal changes. Multimodal imaging helps establish an appropriatediagnosis in these eyes and may offer newer insights into clinical diversity in the presentation and pathogenesis of the disease process.

\section{Conflict of interests}

The authors declare that there is no conflict of interests.

\section{Data availability statement}

The data that support the findings of this study are available from the corresponding author upon reasonablerequest.

Funding

None.

Study association

This study is not associated with any thesis or dissertation work. 


\section{References}

1.Bajgai P, Katoch D, Dogra MR, et al. Idiopathic retinal vasculitis, aneurysms, and neuroretinitis (IRVAN) syndrome: clinical perspectives. Clin Ophthalmol 2017;11:1805-1817.

2. MacIver S, Bass SJ, Sherman J. Visual acuity recovery in a case of idiopathic retinal vasculitis aneurysms and neuroretinitis. Optom Vis Sci 2012;89(3):E356-E363.

3. Chawla R, Kumar A, Ravani R, et al. Multimodal imaging questions etiology of idiopathic retinal vasculitis, aneurysms and neuroretinitis syndrome (IRVAN syndrome). Med Hypotheses 2018;111:12-14.

4. Kumawat D, Kumar V. Resolution of arterial aneurysms in idiopathic retinal vasculitis, aneurysms and neuroretinitis: a case report and review of literature. Int Ophthalmol 2019;39(5):1155-1161.

5. Khan HA, Mehmood A, Khan QA, et al. A major review of optical coherence tomography angiography. Expert Rev Ophthalmol 2017;12(5):373-385.

6. Liu XC, Zhang MN, Chen B, et al. A new perspective for analyzing clinical characteristics of idiopathic retinal vasculitis, aneurysms, and neuroretinitis syndrome. Int Ophthalmol 2019;39(7):1475-1482.

7.Karasu B, Ozkan D, Erdogan G, et al. The fluorescein angiographic photodiagnosis of idiopathic retinal vasculitis, aneurysms, and neuroretinitis (IRVAN) syndrome: Outcome of combined therapy. Photodiagnosis Photodyn Ther 2019;27:336-339.

8. Marchese A, Miserocchi E, Modorati G, et al. Widefield OCT Angiography ofIdiopathic Retinal Vasculitis, Aneurysms, and Neuroretinitis. Ophthalmology Retina 2017;1(6):567-569.

9. Orellana-Rios J, Hussnain SA, Yannuzzi LA. Multimodal Imaging and OCTA of aMacroaneurysm in IRVAN Syndrome. Ophthalmol Retina 2018;2(11):1170.

10.Yeshurun I, Recillas-Gispert C, Navarro-Lopez P, et al. Extensive Dynamics in Location, Shape, and Size of Aneurysms in a Patient With Idiopathic Retinal Vasculitis, Aneurysms, and Neuroretinitis (IRVAN) Syndrome. Am J Ophthalmol 2003;135(1):118-120.

How to cite this article: Ali Khan.H., Shahzad.A.M,

Amer Awan .M. A typical features in IRVAN syndrome

demonstrated on multimodal imaging. Ophthalmology

Cases \& Hypotheses. 2021;02/01:5-9.

Copyright @2021. All rights reserved. 